\title{
Expanding Character Sampling for Ciliate Phylogenetic Inference Using Mitochondrial SSU-rDNA as a Molecular Marker
}

\author{
Micah Dunthorn \\ University of Massachusetts Amherst \\ Wilhelm Foissner \\ Universitat Salzburg \\ Laura A. Katz \\ Smith College, lkatz@smith.edu
}

Follow this and additional works at: https://scholarworks.smith.edu/bio_facpubs

Part of the Biology Commons

\section{Recommended Citation}

Dunthorn, Micah; Foissner, Wilhelm; and Katz, Laura A., "Expanding Character Sampling for Ciliate Phylogenetic Inference Using Mitochondrial SSU-rDNA as a Molecular Marker" (2011). Biological Sciences: Faculty Publications, Smith College, Northampton, MA.

https://scholarworks.smith.edu/bio_facpubs/123

This Article has been accepted for inclusion in Biological Sciences: Faculty Publications by an authorized administrator of Smith ScholarWorks. For more information, please contact scholarworks@smith.edu 


\title{
Expanding Character Sampling for Ciliate Phylogenetic Inference Using Mitochondrial SSU-rDNA as a Molecular Marker
}

\author{
Micah Dunthorn ${ }^{\mathrm{a}, 1}$, Wilhelm Foissner ${ }^{\mathrm{b}}$, and Laura A. Katz ${ }^{\mathrm{a}, \mathrm{c}}$ \\ aProgram in Organismic and Evolutionary Biology, University of Massachusetts, Amherst, MA \\ 01003, USA \\ bFB Organismische Biologie, University of Salzburg, AT-5020 Salzburg, Austria \\ 'Department of Biological Sciences, Smith College, Northampton, MA 01063, USA
}

\section{Abstract}

\begin{abstract}
Molecular systematics of ciliates, particularly at deep nodes, has largely focused on increasing taxon sampling using the nuclear small subunit rDNA (nSSU-rDNA) locus. These previous analyses have generally been congruent with morphologically-based classifications, although there is extensive non-monophyly at many levels. However, caution is needed in interpreting these results as nSSU-rDNA is just a single molecular marker. Here the mitochondrial small subunit rDNA (mtSSU-rDNA) is evaluated for deep ciliate nodes using the Colpodea as an example. Overall, well-supported nodes in the mtSSU-rDNA and concatenated topologies are well supported in the nSSU-rDNA topology; e.g., the non-monophyly of the Cyrtolophosidida. The two moderately-to well-supported incongruences between the loci are the placement of the Sorogenida and Colpoda aspera. Our analyses of mtSSU-rDNA support the conclusion, originally derived from nSSU-rDNA, that the morphological characters used in taxonomic circumscriptions of the Colpodea represent a mixture of ancestral and derived states. This demonstration of the efficacy of the mtSSU-rDNA will enable phylogenetic reconstructions of deep nodes in the ciliate tree of life to move from a single-locus to a multi-locus approach.
\end{abstract}

\section{Keywords \\ Ciliophora; Colpodea; mitochondrial SSU-rDNA; nuclear SSU-rDNA; phylogeny}

\section{Introduction}

Whether it is better to increase the number of sampled taxa or the number of characters to improve the accuracy of phylogenetic inference is a central debate in molecular systematics (Cummings and Meyer 2005; Graybeal 1998; Hedtke et al. 2006; Hillis 1998; Hillis et al. 2003; Poe and Swofford 1999; Rannala et al. 1998; Rokas and Carroll 2005; Rokas et al. 2003). Each approach has its strengths and weaknesses, and it is generally advantageous to increase both when inferring the tree of life of any group of organisms. But this has not always been possible in all clades_-such as in ciliates (Ciliophora Doflein, 1901).

In ciliates, molecular phylogenetic inferences to test morphologically-based hypotheses of deep relationships have relied primarily on expanding taxon sampling using just the nuclear

\footnotetext{
(c) 2010 Elsevier GmbH. All rights reserved.

${ }^{1}$ Corresponding author and present address: Department of Ecology, University of Kaiserslautern, 67653 Kaiserslautern, Germany; fax +496312052496 dunthorn@ @hrk.uni-kl.de. .
} 
small subunit ribosomal DNA (nSSU-rDNA) locus (e.g., Agatha and Strüder-Kypke 2007; Dunthorn et al. 2008, 2009; Gong et al. 2009; Schmidt et al. 2007a, 2007b; Strüder-Kypke et al. 2006; Williams and Clamp 2007; Yi et al. 2008). For these deep ciliate nodes, nSSUrDNA gene trees are concordant with many morphological hypotheses, but there are a number of discrepancies (Dunthorn and Katz 2008; Foissner et al. 2004; Lynn, 2003, 2008). Because of this single-locus approach, we do not know if nSSU-rDNA molecules are elucidating ciliate evolution or just misleading us.

As additional molecular markers, nuclear protein-coding loci are problematic because their extensive paralogy and heterogeneous rates of evolution can lead to spurious phylogenetic relationships (Israel et al. 2002; Katz et al. 2004; Zufall and Katz 2007; Zufall et al. 2006). Moreover, the genome architecture of some ciliates enables generation of macronuclear protein families from alternatively processed and scrambled micronuclear chromosomes (Katz and Kovner 2010), processes that will further confound phylogenetic inferences. In contrast, for shallower ciliate nodes there are a number of available molecular markers from both nuclear protein-coding loci (Catania et al. 2008; Przyboś et al. 2006; Snoke et al. 2006; Ye and Romero 2002) and mitochondrial loci (Barth et al. 2008; Catania et al. 2008; Chantangsi and Lynn 2008; Chantangsi et al. 2007; Gentekaki and Lynn 2009; Lynn and Strüder-Kypke 2006; Snoke et al. 2006; Strüder-Kypke and Lynn 2010).

One ciliate lineage in which nSSU-rDNA genealogies have been compared to morphological hypotheses is the Colpodea Small \& Lynn, 1981 (Figs 1-6). The Colpodea is diagnosed by a LKm (left kinetodesmal) fiber and unique silverline patterns (Foissner 1993; Lynn 2008). This primarily terrestrial group contains diverse oral morphologies and potentially arose 900 MYA (Lynn 2008; Wright and Lynn 1997). The Colpodea may or may not be an ancient asexual clade (Dunthorn et al. 2008; Dunthorn and Katz 2010; Foissner 1993). The almost 200 described species are monographed with an extensive morphological classification (Foissner 1993). Nuclear SSU-rDNA analyses have challenged some aspects of this morphologically-based classification (Dunthorn et al. 2008, 2009; Foissner and Stoeck 2009; Lasek-Nesselquist and Katz 2001; Lynn et al. 1999). In light of the nSSU-rDNA data, modified hypotheses of morphological evolution have been proposed (Dunthorn et al. 2008, 2009; Foissner and Kreutz 1998; Lasek-Nesselquist and Katz 2001; Lynn et al. 1999).

Here we move molecular systematics for deep ciliate nodes towards increasing character sampling by sequencing a broad sample of the Colpodea for another molecular marker: the mitochondrial small subunit rDNA (mtSSU-rDNA) locus. We test if well-supported nodes in the SSU-rDNA topology are congruent with well-supported nodes in the nSSU-rDNA topology, as well as if mtSSU-rDNA provides more resolution in nodes that are unsupported in the nSSU-rDNA topology. Our approach generates additional molecular characters for analyses that are not only from an independent locus but also from a separate genome. Hence, analyzing both nSSU-rDNA and mtSSU-rDNA has the potential to substantially increase our power for inferring deep nodes in the ciliate tree of life and mapping morphological changes within this microbial eukaryotic clade.

\section{Results}

\section{Characteristics of Gene Sequences}

Twenty-one morphospecies were sequenced here for the mtSSU-rDNA locus (Table 1). The amplified products are of variable size and G-C content. For all sequences, the average number of base pairs is 1070, with a minimum of 894 in Chilodonella uncinata and a maximum of 1152 in Colpoda magna. Towards the five-prime end there is considerable variation in length due to insertions and deletions. The average G-C content is $32.9 \%$. 
Intra-isolate genetic variation in the mtSSU-rDNA locus was not found, except in Colpoda henneguyi (Table 1). The distance between the two $C$. henneguyi sequences is $2.69 \%$; this same isolate had two different nSSU-rDNA sequences with a distance $0.12 \%$ (Dunthorn et al. 2008). As the DNA was extracted from a non-clonal culture, these differences may be due to within population variation (or presence of cryptic species) as opposed to within individual variation. The distance between two Cyrtolophosis mucicola isolates-one from Austria, the other from Brazil-is 10.05\%; while their distance in nSSU-rDNA is $1.71 \%$ (Dunthorn et al. 2008). The nSSU-rDNA data from Dunthorn et al. (2008) and the mtSSUrDNA here suggest that these two $C$. mucicola isolates may represent cryptic species.

\section{Mitochondrial SSU-rDNA Analyses}

Ambiguously aligned positions were removed in two ways. First, by eye in MacClade (Maddison and Maddison 2005), resulting in an alignment that includes 823 unmasked characters, of which 491 are parsimony-informative. Second, with the program Gblocks (Castresana 2000; Talavera and Castresana 2007) set to default parameters, resulting in an alignment that includes 731 unmasked characters, of which 410 are parsimony-informative. Initial maximum likelihood analyses using the GTR-I- $\Gamma$ model of evolution showed that the resulting topologies were congruent (data not shown); therefore, the alignment masked by eye was used for further phylogenetic analyses. Bayesian inference was first performed using the GTR-I- $\Gamma$ model of evolution as implemented in MrBayes (Huelsenbeck and Ronquist 2003); here-after referred to as the MrBayes tree.

There is little difference among the MP, ML, and MrBayes topologies for well-supported nodes, and the ML and MrBayes trees are identical. Here we present the most likely ML tree with node support from all three methods (Fig. 7). In all analyses the Colpodea is monophyletic, with moderate to full node support (MP bootstrap 88/ML bootstrap 88/ MrBayes posterior probability 1.00). However, mtSSU-rDNA does not provide a valid test of monophyly here because of the current limited taxon sampling of outgroup lineages.

Mitochondrial SSU-rDNA does not support the monophyly of the Cyrtolophosidida (Fig. 7). The Cyrtolophosidida falls into two clades with moderately supported intervening nodes (78/74/0.95): Cyrtolophosidida I, which includes those taxa in the Cyrtolophosididae, is sister to the Colpodida with no (i.e., < 50) to low node support (-/-/0.78); and Cyrtolophosidida II, which includes the remaining sampled Cyrtolophosidida. Sorogenida is monophyletic with no to low node support (-/54/0.68), and is sister to Cyrtolophosidida II with high to full node support (99/100/1.00). Bryometopida and Bursariomorphida are sister to each other with high to full node support (99/100/1.00). Colpodida is monophyletic in the mtSSU-rDNA topology, though with no to moderate node support (-/72/0.92). Within the shallow ciliate nodes in the Colpodida, Colpoda is not monophyletic as Bresslauides nests within it with high to full node support (90/99/1.00). Both Bardeliella and Hausmanniella also nest within Colpoda in the mtSSU-rDNA topology but with only no to moderate node support (-/69/0.87 and 64/71/0.94). Colpoda aspera is sister to the clade formed by Bardeliella, Bresslauideus, Hausmanniella, and the other sampled Colpoda with no to moderate node support (-/72/0.92).

To account for the possibility of model and rate variation, the mtSSU-rDNA alignment was also analyzed using a second method of Bayesian inference that used a Dirichlet processes of different GTR matrices for a model of evolution as implemented in PhyloBayes (Lartillot and Philippe 2004; Lartillot et al. 2009); hereafter referred to as the PhyloBayes tree. The PhyloBayes tree is largely congruent with the ML and MrBayes tree for relationships within the Colpodea (Fig. 8). The PhyloBayes tree differs in: Sorogenida does not form a resolved clade; and $C$. aspera forms a clade with the remaining Colpoda plus Bresslauideus, although node support is low for this relationship ( 0.56 posterior probability). 


\section{Nuclear SSU-rDNA Analyses}

To test whether truncated taxon sampling will affect the topology of the Colpodea, taxon inclusion in nSSU-rDNA alignment from Dunthorn et al. (2009) was decreased to the same sampling as that of mtSSU-rDNA here. This alignment includes 1631 characters, of which 438 are parsimony-informative. The MP, ML and MrBayes topologies are identical, except that in the ML tree $C$. aspera and $H$. discoidea are sister to each other, but there is no node support for this relationship. Here we present the most likely ML tree with node support from all three methods of analysis (Fig. 9).

The nSSU-rDNA topology for the Colpodea here (Fig. 9) is the same as those previously published analyses based on larger taxon sampling (Dunthorn et al. 2008, 2009), except for the low MP and MrBayes, but moderate ML, node support for the clade formed by Cyrtolophosidida I and Colpodida (57/90/0.57). The low support for this same clade in the $\mathrm{mtSSU}$ topologies (Figs 7, 8), may be due to the lower taxon sampling here, and may increase as more taxa are sampled for the mtSSU-rDNA.

\section{Topology Testing}

Overall, the nSSU- and the mtSSU-rDNA topologies are congruent for well-supported nodes, except in the placement of the Sorogenida and $C$. aspera. To further compare the loci, Approximately Unbiased (AU) tests were carried out on the mtSSU-rDNA alignment where the ambiguously aligned positions were removed by eye, the mtSSU-rDNA alignment where the positions were removed by GBlock, and on the nSSU-rDNA alignment (Table 2).

In the mtSSU-rDNA topology the Sorogenida is sister to the Cyrtolophosidida II (Fig. 7), while in the nSSU-rDNA topology the Sorogenida is sister only to Platyophrya bromelicola (Fig. 9). The mtSSU-rDNA alignments could not reject the constrained topology where the Sorogenida was forced to be sister to P. bromelicola, as in the nSSU-rDNA topology ( $\mathrm{p}=$ 0.159 and 0.283 ). Likewise, the nSSU-rDNA topology could not reject the constrained topology where the Sorogenida was forced to be sister to the clade formed by Platyophryal Sagittarial Rostrophryal Platyophrya-like, as in the mtSSU-rDNA topology $(\mathrm{p}=0.365)$. Therefore, the phylogenetic placement of the Sorogenida remains ambiguous.

In the mtSSU-rDNA topology $C$. aspera is sister to the clade formed by Bardeliella, Hausmanniella, Bresslauides, and the remaining Colpoda (Fig. 7), while in the nSSU-rDNA topology C. aspera is sister to just Hausmanniella (Fig. 9). All alignments were able to reject the constraint that all Colpoda form a monophyletic clade, as in the morphological classification $(\mathrm{p}=0.005,0.035,0.009)$. However, the alignments were not able to reject the constraint that all Colpoda plus Bresslauides form a monophyletic clade $(\mathrm{p}=0.371,0.521$, 0.572). Therefore, the phylogenetic placement of $C$. aspera remains ambiguous at least in relation to Bardeliella and Hausmanniella.

\section{Concatenated Analyses}

Given the overall congruence between the topologies and the results of the AU tests, a concatenated alignment of the nSSU- and mtSSU-rDNA sequences was compiled in order to further evaluate phylogenetic relationships in the Colpodea. This alignment includes 2454 characters, of which 929 are parsimony-informative. There was little difference in the MP, ML, and MrBayes topologies for well-supported nodes, and the ML and MrBayes trees are identical. Here we present the most likely ML tree with node support from all three methods (Fig. 10).

The nSSU-rDNA, mtSSU-rDNA, and concatenated topologies are largely congruent with each other for almost all relationships, except for the placement of Sorogenida and C.aspera 
(Figs 7-10). Like the mtSSU-rDNA topology (Fig. 7), Sorogenida is sister to Cyrtolophosidida II in the concatenated topology (Fig. 10). Colpoda aspera forms clade with the remaining Colpoda and Bresslauides with no node support (-/-/-). The clade formed by Cyrtolophosidida I and Colpodida has low MP and MrBayes, but moderate ML, node support in the concatenated topology (-/84/0.87). This low MP and MrBayes support may likewise be due to low taxon sampling (see above).

To account for the possibility of model and rate variation, the concatenated alignment was likewise analyzed using the Dirichlet processes of different GTR matrices in PhyloBayes (Lartillot and Philippe 2004; Lartillot et al. 2009). The PhyloBayes tree is largely congruent with the ML and MrBayes tree for relationships within the Colpodea (Fig. 11). The PhyloBayes tree differs in that the Sorogenida forms a paraphyletic lineage at the base of the Cyrtolophosidida II.

\section{Discussion}

\section{Phylogenetic Relationships in the Colpodea}

The potential problem that individual gene trees may not necessarily reflect the species tree affects all organisms (Doyle 1992, 1997; Maddison 1997). So in ciliates there have been critiques that nSSU-rDNA gene trees may not provide an accurate inference of phylogeny where there are discrepancies between data from morphology and molecules (Agatha 2004; Dunthorn et al. 2008; Foissner et al. 2004; Schmidt et al. 2007a). On the other hand, alternative hypotheses, or re-interpretations, of morphological evolution given the topology of nSSU-rDNA gene trees have been suggested (Dunthorn et al. 2008; Dunthorn and Katz 2008; Lynn et al. 1999; Strüder-Kypke and Lynn 2003). To help resolve this, here we present additional and independent molecular data from the mtSSU-rDNA locus for testing hypotheses of relationships and morphological evolution for deep ciliate nodes.

When there are discrepancies between morphology and nSSU-rDNA analyses within the Colpodea for deep nodes, the mtSSU-rDNA genealogy largely supports inferences made from analyses of nSSU-rDNA. For example, in our mtSSU-rDNA (Figs 7, 8), nSSU-rDNA (Fig. 9), the concatenated (Figs 10,11) analyses, as well as those from previous nSSUrDNA analyses (Dunthorn et al. 2008, 2009), the Cyrtolophosidida falls out into two separate clades separated by moderate to well-supported nodes: Cyrtolophosidida I and Cyrtolophosidida II. These two Cyrtolophosidida groups differ in details of their oral structures (Dunthorn et al. 2008; Foissner et al. 2002; Figs 4, 5).

Bryometopida and Bursariomorphida form a clade in analyses of nSSU-rDNA (Dunthorn et al. 2008; Lynn et al. 1999; Fig. 9). The mtSSU-rDNA (Figs 7, 8) and concatenated (Figs 10, 11) topologies support this relationship with high to full node support. Dunthorn et al. (2008), Foissner and Kreutz (1998), and Lynn et al. (1999) note that the Bryometopida and Bursariomorphida do share a number of morphological characters: apical oral structures, ventral clefts, adoral organelles that are conspicuous, and cysts with emergence pores.

Colpodida and Cyrtolophosidida I also form a clade in both the current analysis and previous nSSU-rDNA analyses (Dunthorn et al. 2008, 2009; Fig. 9). The mtSSU-rDNA (Figs 7,8) and concatenated (Figs 10,11) topologies likewise support this relationship with no to moderate node support. There is currently no known morphological character that unites these two groups, as the Cyrtolophosidida I may retain the ancestral morphological condition of the Colpodea, while the Colpodida exhibits numerous derived morphologies (Dunthorn et al. 2008). 
Not all relationships are congruent between nSSU-rDNA and mtSSU-rDNA for deep nodes. The Sorogenida nests within the Cyrtolophosidida II in the current and previous nSSUrDNA analyses with no to full node support (Dunthorn et al. 2008, 2009; Fig. 9). In contrast, the mtSSU-rDNA (Figs 7, 8) and the concatenated (Figs 10, 11) analyses place the Sorogenida sister to the Cyrtolophosidida II, likewise with no to full node support. Some possible explanations for this incongruence are differential rates of evolution between the loci or incomplete lineage sorting. In theAU tests, the unconstrained nSSU-rDNA topology could not reject the constrained topology that matched the mtSSU-rDNA relationship; likewise the unconstrained mtSSU-rDNA topologies could not reject the constrained topology, following the nSSU-rDNA relationship (Table 2). The phylogenetic placement of the Sorogenida thus remains unresolved. Although additional taxon sampling of previously unsequenced Cyrtolophosidia II species is needed to resolve the position of these taxa, at least a close relationship between Sorogenida and Cyrtolophosidida II is supported in that both having brick-shaped organelles on the left side of the oral structure as well as pleurotelokinetal stomatogenesis (partial re-organization of parental oral structures during cell division) (Dunthorn et al. 2008).

Within the shallow relationships in the Colpodida, node support is variable and there is a lack of resolution for many relationships from both molecular markers (Figs 1-3, 6-11). Colpoda is not monophyletic as Bresslauides nests within in the nSSU-rDNA, mtSSUrDNA, and concatenated topologies here with high to full node support (Figs 7-11) and in a previous nSSU-rDNA analysis (Dunthorn et al. 2008). The non-monophyly of Colpoda in relation to Bresslauides is also supported by AU tests of both loci in that the topologies could reject the constraint that only the Colpoda isolates form a monophyletic clade (Table 2). Dunthorn et al. (2008) suggest that Bresslauides was taxonomically split off from Colpoda because of potential apomorphies (e.g., large semicircular right oral polykinetids, larger size, and feeding on other ciliates) that arose from within the Colpoda clade (Figs 1$3)$.

It should be noted that Bresslauides is not sister to the other sampled member of the Hausmanniellidae (i.e., Hausmanniella), suggesting that either the diagnostic characters for the Hausmanniellidae arose convergently or that the independent nSSU- and mtSSU-rDNA markers are both misleading in the same way. Although Colpoda henneguyi, C. cucullus (Fig. 4), and C. lucida are morphologically so similar that they were separated only recently (Foissner 1993); however, in the mtSSU-rDNA topology (Fig. 7) C. henneguyi is sister to Bresslauides. This relationship may be unlikely given that Bresslauides is much larger than the three Colpoda (200 - 600 $\mu \mathrm{m}$ vs. 60-150 $\mu \mathrm{m}$ ) and has a different lifestyle (eats other ciliates vs. bacteriovorous). Either both nSSU- and mtSSU-rDNA are misleading at this depth in the ciliate tree of life, or morphological evolution in the Colpoda may be extremely fast, resulting in multiple convergent morphologies.

In the current and previous analyses using nSSU-rDNA (Dunthorn et al. 2008, 2009; Fig. 9), C. aspera is sister to Hausmanniella with no node support. The mtSSU-rDNA topology from MP, ML, and MrBayes analyses (Fig. 7) places $C$. aspera basal to Hausmanniella and Bardeliella with no to moderate node support. This is an odd placement for $C$. aspera given that its horizontally oriented left oral ciliary field is quite unlike those in Bardeliella and Hausmanniella (Figs 2, 3, 6). However, in the PhyloBayes tree (Fig. 8) and in the concatenated analyses (Figs 10,11), C. aspera forms a clade with the remaining Colpoda plus Bresslauides with no to full node support. Furthermore, in the AU tests the mtSSUrDNA and the nSSU-rDNA alignments could not reject the monophyletic clade formed by Colpoda and Bresslauides (Table 2). The phylogenetic placement of $C$. aspera thus remains ambiguous. 
The rate of substitution appears to be faster in mtSSU-rDNA than in nSSU-rDNA, such as in $C$. henneguyi and C. mucicola. This discrepancy in rates can be explained by a number of possible factors: smaller effective population size of the nuclei vs. mitochondria; homogenizing effects on nSSU-rDNA due to meiotic recombination, although there is debate as to whether the Colpodea are sexual (Dunthorn et al. 2008; Dunthorn and Katz 2010; Foissner 1993); elevated rates of mutations in the mitochondrial genome; and/or differing levels of functional constraints between the SSU-rDNAs of the two genomes.

\section{Mitochondrial SSU-rDNA As a Ciliate Molecular Marker}

Nuclear SSU-rDNA has remained the primary locus for molecular phylogenetic inferences of deep ciliate nodes since it was first sequenced by Sogin and Elwood (1986) and Lynn and Sogin (1988). Although congruent in many aspects with morphologically-based classifications, nSSU-rDNA topologies have been used to break up or reshuffle large taxa, as well as recognize new clades (Affa'a et al. 2004; Gong et al. 2009; Greenwood et al. 1991; Lynn 2003, 2008; Lynn and Strüder-Kypke 2002; Stoeck et al. 2007; Strüder-Kypke et al. 2006; Strüder-Kypke and Lynn 2003; van Hoek et al. 2000b; Yi et al. 2008). The resulting reliance on just nSSU-rDNA to infer deep nodes in the ciliate tree of life stands in contrast to the increasing repertoire of both low- and high-copy loci available for many other microbial and macro-organismic eukaryotic clades, as well as the number of loci used to reconstruct relationships within and among closely related ciliate species.

Here we show that the mtSSU-rDNA locus can infer well-supported nodes for the depths of the ciliate tree of life that were analyzed (i.e., in the Colpodea). Furthermore, most of the nodes in the individual (Figs 7,8) and concatenated (Figs 10,11) analyses are congruent with those that are well supported in previous nSSU-rDNA analyses (Dunthorn et al. 2008, 2009; Foissner and Stoeck 2009; Lasek-Nesselquist and Katz 2001; Lynn et al. 1999), as well as the truncated taxon sampling here (Fig. 9). We did not find that mtSSU-rDNA provides more resolution than nSSU-rDNA, as unsupported nodes remained unsupported in the Colpodea.

Future molecular phylogenetic inferences of deep nodes in the ciliate tree of life can now use a two-locus approach — with the nuclear and mitochondrial SSU-rDNA. This increasing of character sampling will help bring ciliate molecular systematics up to current practices in other eukaryotic clades where the use of multiple, independent molecular markers is both standard and expected. While we provide a two locus-approach for deep ciliate nodes in the Colpodea, in other eukaryotic clades it has been shown that the use of many more molecular markers drastically improves phylogenetic resolution (Philippe et al. 2005; Rokas et al. 2003). We anticipate that phylogenomic analyses are on the horizon for ciliate systematics, particularly for those lineages that can be cultured to obtain sufficient amounts of RNA for transcriptome analyses. At the same time, analysis of both nSSU-rDNA and mtSSU-rDNA will remain a powerful approach for those wishing to survey large numbers of taxa and/or those starting with limited numbers of cells.

\section{Methods}

\section{Taxon sampling and terminology}

Sequences were obtained from genomic DNA from earlier phylogenetic studies (Dunthorn et al. 2008, 2009; Riley and Katz 2001), as well as from GenBank. In total, our sampling includes 25 isolates from 24 morphospecies for mtSSU-rDNA (Table 1). One of us (W.F.) provided and identified most of the species used in this study. Of these, 20 are from the Colpodea. Exemplars from five of the seven orders within the Colpodea as recognized by Foissner (1993) are in included (Figs 1-6). For nSSU-rDNA, we started with the alignment 
from Dunthorn et al. (2009). Two Paramecium species, two Tetrahymena species, and Chilodonella uncinata are included as outgroups. Initial analyses included mtSSU-rDNA hydrogenosome sequences from Armorphorea accessions in GenBank; these were excluded from the final analyses since they exhibited extreme rate heterogeneity compared to the rest of the sequences (possibly due to the evolution from mitochondria to hydrogenosome). When possible, both nSSU-rDNA and mtSSU-rDNA were from the same source DNA. Terminology follows Foissner (1993) and Lynn (2008). Classification follows Foissner (1993), with the addition of the labelling of Cyrtolophosidida clades 1 and 2 in the trees following Dunthorn et al. (2008).

\section{DNA amplification and sequencing}

Genomic DNA was extracted using the DNeasy Tissue kit (Qiagen, CA). Mitochondrial SSU-rDNA was amplified with the $5^{\prime}$ primer (TGT GCC AGC AGC CGC GGT AA) and the $3^{\prime}$ primer (CCC MTA CCR GTA CCT TGT GT) from van Hoek et al. (2000a). Phusion polymerase (New England BioLabs, MA) was used with the following cycling conditions: 3:00 at $98^{\circ} \mathrm{C} ; 40$ cycles of $0: 15$ at $98^{\circ} \mathrm{C}, 0: 15$ at $67{ }^{\circ} \mathrm{C}, 1: 15$ at $72{ }^{\circ} \mathrm{C} ; 10: 00$ extension at 72 ${ }^{\circ} \mathrm{C}$.

Amplified products were cleaned with microCLEAN (The Gel Company, San Francisco, $\mathrm{CA}$ ), and cloned with the Zero Blunt TOPO kit (Invitrogen, Carlsbad, CA). Positive clones were identified by PCR screening with AmpliTag Gold polymerase and vector primers (Applied Biosystems, Foster City, CA), and minipreped using Qiaprep Spin Miniprep kit (Qiagen). Clones were sequenced with the Big Dye terminator kit (Applied Biosystems), using vector primers. Up to eight colonies were sequenced in the forward direction; up to five of these were also sequenced in the reverse direction. Sequences were run on an ABI 3100 automated sequencer.

\section{Alignments and removal of ambiguous positions}

Mitochondrial sequences were determined and edited from overlapping sequence reads in SeqMan (DNAStar, Inc., Madison, WI). Vector and primer nucleotides were trimmed off. Sequences were aligned using Clustal X (Thompson et al. 1994), and further edited by eye in MacClade v4.05 (Maddison and Maddison 2005). Removal of ambiguously aligned positions was performed in two different ways: by eye in MacClade, and using Gblocks v0.91b (Castresana 2000; Talavera and Castresana 2007) set to default parameters (min conserved $=13$, min flank $=21$, $\max$ nonconserved $=8$, min block $=10$, gap $=$ none) . The two resulting alignments were analysed separately. For the nSSU-rDNA, the taxon sampling in the alignment from Dunthorn et al. (2009) was truncated so that it included the same taxa as the mtSSU-rDNA alignment.

\section{Genealogical analyses}

Pairwise distances for within and among samples were calculated as uncorrected "p" distances in PAUP* v4.0b8 (Swofford 2002). For all datasets the GTR-I- $\Gamma$ evolutionary model was the best fitted model selected by AIC in MrModeltest v2 (Nylander 2004). Maximum parsimony (MP) analyses were carried out in PAUP* v4.0b8 (Swofford 2002), with all characters equally weighted and unordered. The TBR heuristic search option was used, running 100 random additions with MulTree option on, and support came from 1000 bootstrap replicates. Maximum likelihood (ML) analyses were carried out in RaxM-HPC v7.2.5 (Stamatakis et al. 2008). Support came from a majority rule consensus tree of 1000 multiparametic bootstrap replicates carried out in RaxM-HPC.

Bayesian Inference (BI) was carried out using two different algorithms. First with MrBayes v3.2.1 (Huelsenbeck and Ronquist 2003) using the GTR-I-Г model. Posterior probability 
was estimated using four chains running 10 million generations sampling every 1000 generations. To determine if Bayesian analyses were run long enough, output files were examined using AWTY (Nylander et al. 2008). Second, to account to the possibility of model and rate variation, PhyloBayes v3.2e (Lartillot and Philippe 2004; Lartillot et al. 2009) was used with the QMM model (Dirichlet processes of GTR matrices). Posterior probability was estimated using one chain running at least 1.5 million generations sampling every cycle. For both methods the first $25 \%$ of sampled trees were considered burn-in trees and were discarded prior to constructing a 50\% majority rule consensus trees. Trees were visualized with FigTree v1.3.1 (Rambaut 2006).

\section{Topology testing}

Three constrained ML analyses were carried out on the mtSSU-rDNA alignment masked by eye and the alignment masked using GBlocks: Ottowphrya + Platyophrya + Sorogena monophyletic, as in the nSSU-rDNA topology; Colpoda monophyletic, as in the morphological classification; and Colpoda + Bresslauides monophyletic. Resulting constrained topologies were then compared to the non-constrained ML topology using the AU test (Shimodaira 2002) as implemented in CONSEL v0.1j (Shimodaira and Hasegawa 2001). Likewise, three constrained ML analyses were carried out on the nSSU-rDNA dataset: Platyophrya + Platyophrya-like + Rostrophrya + Sagittaria monophyletic, as in the mtSSU-rDNA topology; Colpoda monophyletic, as in the morphological classification; and Colpoda + Bresslauides monophyletic. Resulting constrained topologies were then compared to the non-constrained ML topology using the AU test. For all constrains, internal relationships within the constrained groups was unspecified, and relationships among the remaining taxa were unspecified as well.

\section{Acknowledgments}

We would like to thank Mark Beilstein, Jessica Grant, Dan Lahr, Rob Dorit, George McManus, Ben Normark, Hervé Philippe, Thorsten Stoeck, Patrick Sweeney, and two anonymous reviewers for comments and suggestions. This work was supported by postdoctoral fellowships from the Faculty of Biology of the University of Kaiserslautern and the Alexander von Humboldt Foundation to M.D., the Austrian Science Foundation (projects P-20360B17 and P-19699-B17) to W.F., and the United States National Science Foundation (grant DEB 0816828) to L.A.K.

\section{References}

Affa'a FM, Hickey DA, Strüder-Kypke M, Lynn DH. Phylogenetic position of species in the genera Anoplophrya, Plagiotoma, and Nyctotheroides (Phylum Ciliophora), endosymbiotic ciliates of annelids and anurans. J Eukaryot Microbiol. 2004; 51:301-306. [PubMed: 15218698]

Agatha S. Evolution of ciliary patterns in the Oligotrichida (Ciliophora, Spirotricha) and its taxonomic implications. Zoology. 2004; 107:153-168. [PubMed: 16351935]

Agatha S, Strüder-Kypke M. Phylogeny of the order Choreotrichida (Ciliophora, Spriotricha, Oligotrichea) as inferred from morphology, ultrastructure, ontogenesis, and SSr-RNA sequences. Eur J Protistol. 2007; 43:37-63. [PubMed: 17166704]

Barth D, Tischer K, Berger H, Schlegel M, Berendonk TU. High mitochondrial haplotype diversity of Coleps sp. (Ciliophora: Prostomatida). Environ Microbiol. 2008; 10:626-634. [PubMed: 18190514]

Castresana J. Selection of conserved blocks from multiple alignments for their use in phylogenetic analysis. Mol Biol Evol. 2000; 17:540-552. [PubMed: 10742046]

Catania F, Wurmser F, Potekhin AA, Przyboś E, Lynch M. Genetic diversity in the Paramecium aurelia species complex. Mol Biol Evol. 2008; 26:421-431. [PubMed: 19023087]

Chantangsi C, Lynn DH. Phylogenetic relationships within the genus Tetrahymena inferred from cytochrome c oxidase subunit 1 and the smal subunit ribosomal RMA genes. Mol Phylogenet Evol. 2008; 49:979-987. [PubMed: 18929672] 
Chantangsi C, Lynn DH, Brandl MT, Cole JC, Hetrick N, Ikonomi P. Barcoding ciliates: a comprehensive study of 75 isolates of the genus Tetrahymena. Int J Syst Evol Microbiol. 2007; 57:2412-2425. [PubMed: 17911319]

Cummings MP, Meyer A. Magic bullets and golden rules: data sampling in molecular phylogenetics. Zoology. 2005; 108:329-336. [PubMed: 16351981]

Doyle JJ. Gene trees and species trees: molecular systematics as one-character taxonomy. Syst Bot. 1992; 17:144-163.

Doyle JJ. Trees within trees: genes and species, molecules and morphology. Syst Biol. 1997; 46:537553. [PubMed: 11975332]

Dunthorn M, Katz LA. Richness of morphological hypotheses in ciliate systematics allows for detailed assessment of homology and comparisons with gene trees. Denisia. 2008; 23:389-394.

Dunthorn M, Katz LA. Secretive ciliates and putative asexuality in microbial eukaryotes. Trends Microbiol. 2010; 18:183-188. [PubMed: 20299224]

Dunthorn M, Foissner W, Katz LA. Molecular phylogenetic analysis of class Colpodea (phylum Ciliophora) using broad taxon sampling. Mol Phylogenet Evol. 2008; 48:316-327. [PubMed: 17920937]

Dunthorn M, Eppinger M, Schwarz MVJ, Schweikert M, Boenigk J, Katz LA, Stoeck T. Phylogenetic placement of the Cyrtolophosididae Stokes, 1888 (Ciliophora; Colpodea) and neotypification of Aristerostoma marinum Kahl, 1931. Int J Syst Evol Microbiol. 2009; 59:167-180. [PubMed: 19126743]

Foissner W. Colpodea (Ciliophora). Protozoenfauna. 1993; 4/1:i-x. 1-798.

Foissner W, Kreutz M. Systematic position and phylogenetic relationships of the genera Bursaridium, Paracondylostoma, Thylakidium, Bryometopus, and Bursaria (Ciliophora: Colpodea). Acta Protozool. 1998; 37:227-240.

Foissner W, Stoeck T. Morphological and molecular characterization of a new protist family, Sandmanniellidae n. fam. (Ciliophora, Colpodea), with description of Sandmanniella terricola n. g., n. sp. from the Chobe floodplain in Botswana. J Eukaryot Microbiol. 2009; 56:472-483. [PubMed: 19737201]

Foissner W, Agatha S, Berger H. Soil ciliates (Protozoa, Ciliophora) from Namibia (Southwest Africa), with emphasis on two contrasting environments, the Etosha Region and the Namib Desert. Densia. 2002; 5:1-1459.

Foissner W, Moon-van der Staay SY, van der Staay GWM, Hackstein JHP, Krautgartner W-D, Berger $\mathrm{H}$. Reconciling classical and molecular phylogenies in the stichotrichines (Ciliophora, Spirotrichea), including new sequences from some rare species. Eur J Protistol. 2004; 40:265-281.

Gentekaki E, Lynn DH. High-level genetic diversity bot no population structure inferred from nuclear and mitochondrial markers of the peritrichous ciliate Carchesium polypinum in the Grand River Bason (North America). Appl Environ Microbiol. 2009; 75:3187-3195. [PubMed: 19304815]

Gong J, Stoeck T, YI Z, Miao M, Zhang N, Roberts DM, Warren A, Song W. Small subunit rDNA phylogenies show that the class Nassophorea is not monophyletic (Phylum Ciliophora). J Eukaryot Microbiol. 2009; 56:339-347. [PubMed: 19602079]

Graybeal A. Is it better to add taxa or characters to a difficult phylogenetic problem? Syst Biol. 1998; 47:9-17. [PubMed: 12064243]

Greenwood SJ, Sogin ML, Lynn DH. Phylogenetic relationships within the class oligohymenophorea, phylum Ciliophora, inferred from the complete small subunit rRNA gene sequences of Colpidium campylum, Glaucoma chattoni and Opisthonecta henneguyi. J Mol Evol. 1991; 33:163-174. [PubMed: 1840618]

Hedtke SM, Townsend TM, Hillis DM. Resolution of phylogenetic conflict in large data sets by increased taxon sampling. Syst Biol. 2006; 55:522-529. [PubMed: 16861214]

Hillis DM. Taxonomic sampling, phylogenetic accuracy, and investigator bias. Syst Biol. 1998; 47:38. [PubMed: 12064238]

Hillis DM, Pollock DD, McGuire JA, Zwickl DJ. Is sparse taxon sampling a problem for phylogenetic inference? Syst Biol. 2003; 52:124-126. [PubMed: 12554446]

Huelsenbeck JP, Ronquist FR. MrBayes 3: Bayesian phylogenetic inference under mixed models. Bioinformatics. 2003; 19:1572-1574. [PubMed: 12912839] 
Israel RL, Pond SL Kosakovsky, Muse SV, Katz LA. Evolution of duplicated alpha-tubulin genes in ciliates. Evolution. 2002; 56:1110-1122. [PubMed: 12144013]

Katz LA, Kovner AM. Multiple origins of scrambled coding domains within rapidly evolving germline sequences. J Exp Zool (Mol Dev Evol). 2010; 314B

Katz LA, Lasek-Nesselquist E, Bornstein J, Muse SV. Dramatic diversity of ciliate histone H4 genes revealed by comparisons of patterns of substitutions and paralog divergences among eukaryotes. Mol Biol Evol. 2004; 21:555-562. [PubMed: 14694079]

Lartillot N, Philippe H. A Bayesian mixture model for across-site heterogeneities in the amino-acid replacement process. Mol Biol Evol. 2004; 21:1095-1109. [PubMed: 15014145]

Lartillot N, Lepage T, Blanquart S. PhyloBayes 3: a Bayesian software package for phylogenetic reconstruction and molecular dating. Bioinformatics. 2009; 25:2286-2288. [PubMed: 19535536]

Lasek-Nesselquist E, Katz LA. Phylogenetic position of Sorogena stoianovitchae and relationships within the class Colpodea (Ciliophora) based on SSU rDNA sequences. J Eukaryot Microbiol. 2001; 48:604-607. [PubMed: 11596926]

Lynn DH. Morphology or molecules: how do we identify the major lineages of ciliates (Phylum Ciliophora)? Eur J Protistol. 2003; 39:356-364.

Lynn, DH. The Ciliated Protozoa: Characterization, Classification, and Guide to the Literature. 3rd edn. Springer; Dordrecht: 2008.

Lynn DH, Sogin ML. Assessment of phylogenetic relationships among ciliated protists using partial ribosomal RNA sequences derived from reverse transcripts. Biosystems. 1988; 21:249-254. [PubMed: 2456107]

Lynn DH, Strüder-Kypke M. Phylogenetic position of Licnophora, Lechriopyla, and Schizocaryum, three unusual ciliates (Phylum Ciliophora) endosymbiotic in echinoderms (Phylum Echinodermata). J Eukaryot Microbiol. 2002; 49:460-468. [PubMed: 12503681]

Lynn DH, Strüder-Kypke M. Species of Tetrahymena identical by small subunut rRNA gene sequences are discriminated by mitochondrial cytochrome c oxidase I gene sequences. J Eukaryot Microbiol. 2006; 53:385-387. [PubMed: 16968457]

Lynn DH, Wright ADG, Schlegel M, Foissner W. Phylogenetic relationships of orders within the class Colpodea (phylum Ciliophora) inferred from small subunit rRNA gene sequences. J Mol Evol. 1999; 48:605-614. [PubMed: 10198126]

Maddison WP. Gene trees in species trees. Syst Biol. 1997; 46:523-536.

Maddison, WP.; Maddison, DR. MacClade. v. 4.0.8. Sinauer Assoc.; 2005.

Nylander, JA. MrModeltest v2. Distributed by the Author. Evolutionary Biology Center, Uppsala University; Upsalla: 2004.

Nylander JA, Wilgenbusch JC, Warren DL, Swofford DL. AWTY (are we there yet?): a system for graphical exploration of MCMC convergence in Bayesian phylogenetics. Bioinformatics. 2008; 24:581-583. [PubMed: 17766271]

Philippe H, Delsuc F, Brinkmann H, Lartillot N. Phylogenomics. Annu Rev Ecol Evol Syst. 2005; 36:541-562.

Poe S, Swofford DL. Taxon sampling revisited. Nature. 1999; 398:299-300. [PubMed: 10192331]

Przyboś E, Maciejewska A, Skotarczak B. Relationship of species of the Paramecium aurelia complex (Protozoa, ph. Ciliophora, cl. Oligohymenophorea) based on sequences of the histone $\mathrm{H} 4$ gene fragment. Folia Biol (Praha). 2006; 54:37-42.

Rambaut, A. FigTree. Institute of Evolutionary Biology, Univ. of Edinburgh; 2006. Available at:http:// tree.bio.ed.ac.uk/software/figtree

Rannala B, Huelsenbeck JP, Yang Z, Nielsen R. Taxon sampling and the accuracy of large phylogenies. Syst Biol. 1998; 47:702-710. [PubMed: 12066312]

Riley JL, Katz LA. Widespread distribution of extensive genome fragmentation in ciliates. Mol Biol Evol. 2001; 18:1372-1377. [PubMed: 11420375]

Rokas A, Carroll SB. More genes or more taxa? The relative contribution of gene number and taxon number to phylogenetic accuracy. Mol Biol Evol. 2005; 22:1337-1344. [PubMed: 15746014]

Rokas A, Williams BL, King N, Carroll SB. Genome-scale approaches to resolving incongruence in molecular phylogenies. Nature. 2003; 425:798-804. [PubMed: 14574403] 
Schmidt SL, Bernard D, Schlegel M, Foissner W. Phylogeny of the Stichotrichea (Ciliophora: Spirotrichea) reconstructed with nuclear small subunit rRNA gene sequences: discrepancies and accordances with morphological data. J Eukaryot Microbiol. 2007a; 54:201-209. [PubMed: 17403161]

Schmidt SL, Foissner W, Schlegel M, Bernhard D. Molecular phylogeny of the Heterotrichea (Ciliophora, post-ciliodesmatophora) based on small subunit rRNA gene sequences. J Eukaryot Microbiol. 2007b; 54:358-363. [PubMed: 17669161]

Shimodaira H. An approximately unbiased test of phylogenetic tree selection. Syst Biol. 2002; 51:492508. [PubMed: 12079646]

Shimodaira H, Hasegawa M. CONSEL: for assessing the confidence of phylogenetic tree selection. Bioinformatics. 2001; 17:1246-1247. [PubMed: 11751242]

Snoke MS, Berendonk TU, Barth D, Lynch M. Large global effective population sizes in Paramecium. Mol Biol Evol. 2006; 23:2474-2479. [PubMed: 16987953]

Sogin ML, Elwood HJ. Primary structure of the Paramecium tetraurelia small-subunit rRNA coding region: phylogenetic relationships within the Ciliophora. J Mol Evol. 1986; 23:53-60. [PubMed: 3084799]

Stamatakis A, Hoover P, Rougemont J. A fast boostrap algorithm for the RAxML web-servers. Syst Biol. 2008; 57:758-771. [PubMed: 18853362]

Stoeck T, Foissner W, Lynn DH. Small-subunit rDNA phylogenies sugest that Epalxella antiquorum (Penard, 1922) Corliss, 1960 (Ciliophora, Odontostomatida) is a member of the Plagyopylea. J Eukaryot Microbiol. 2007; 54:436-442. [PubMed: 17910688]

Strüder-Kypke M, Wright A-DG, Foissner W, Lynn DH. Molecular phylogeny of litostome ciliates (Ciliophora, Litostomatea) with emphasis on free-living haptorian genera. Protist. 2006; 157:261278. [PubMed: 16843062]

Strüder-Kypke MC, Lynn DH. Sequence analyses of the small subunit rRNA gene confirm the paraphyly of oligotrich ciliates sensu lato and support the monophyly of the subclasses Oligotrichia and Choreotrichia (Ciliophora, Spirotrichea). J Zool. 2003; 260:87-97.

Strüder-Kypke MC, Lynn DH. Comparative analysis of the mitochondrial cytochrome $c$ oxidase subunit 1 (CO1) gene in ciliates (Alveolata, Ciliophora) and evaluation of its suitability as a biodiversity marker. Syst Biodivers. 2010; 8:131-148.

Swofford, D. PAUP*. Phylogenetic Analysis Using Parsimony (*and Other Methods). Sinauer Assoc; Sunderland MA: 2002.

Talavera G, Castresana J. Improvement of phylogenies after removing divergent and ambiguously aligned blocks from protein sequence alignments. Syst Biol. 2007; 56:564-577. [PubMed: 17654362]

Thompson JD, Higgins DG, Gibson TJ. Clustal W: improving the sensitivity of progressive multiple sequence alignment through sequence weighting, position-specific gap penalties and weight matrix choice. Nucleic Acids Res. 1994; 22:4673-4680. [PubMed: 7984417]

van Hoek AHAM, Akhmanova AS, Huynen MA, Hackstein JHP. A mitochondrial ancestry of the hydrogenosomes of Nyctotherus ovalis. Mol Biol Evol. 2000a; 17:202-206. [PubMed: 10666720]

van Hoek AHAM, van Alen TA, Sprakel VSI, Leunissen JAM, Brigge T, Vogels GD, Hackstein JHP. Multiple acquisitions of methanogenic archaeal symbionts by anaerobic ciliates. Mol Biol Evol. 2000b; 17:251-258. [PubMed: 10677847]

Williams D, Clamp JC. A molecular phylogenetic investigation of Opisthonecta and related genera (Ciliophora, Peritrichia, Sessilida). J Eukaryot Microbiol. 2007; 54:317-323. [PubMed: 17552988]

Wright A-DG, Lynn DH. Maximum ages of ciliate lineages estimated using a small subunit rRNA molecular clock:crown eukaryotes date back to the paleoprotoerozoic. Arch Protistenkd. 1997; 148:329-341.

Ye AJ, Romero DP. Phylogenetic relationships amongst tetrahymenine ciliates inferred by a comparison of telomerase RNAs. Int J Syst Evol Microbiol. 2002; 52:2297-3202. [PubMed: 12508900]

Yi Z, Song W, Warren A, Roberts DM, Al-Rasheid KAS, Chen Z, Al-Farraj SA, Hu X. A molecular phylogenetic investigation of Pseudoamphisiellia and Parabirojima (Protozoa, Ciliophora, 
Spirotrichea), two genera with ambiguous systematic positions. Eur J Protistol. 2008; 44:45-53. [PubMed: 17900880]

Zufall RA, Katz LA. Micronuclear and macronuclear forms of $\beta$-tubulin genes in the ciliate Chilodonella uncinata reveal insights into genome processing and protein evolution. J Eukaryot Microbiol. 2007; 54:275-282. [PubMed: 17552983]

Zufall RA, McGrath CL, Muse SV, Katz LA. Genome architecture drives protein evolution in ciliates. Mol Biol Evol. 2006; 23:1681-1687. [PubMed: 16760419] 

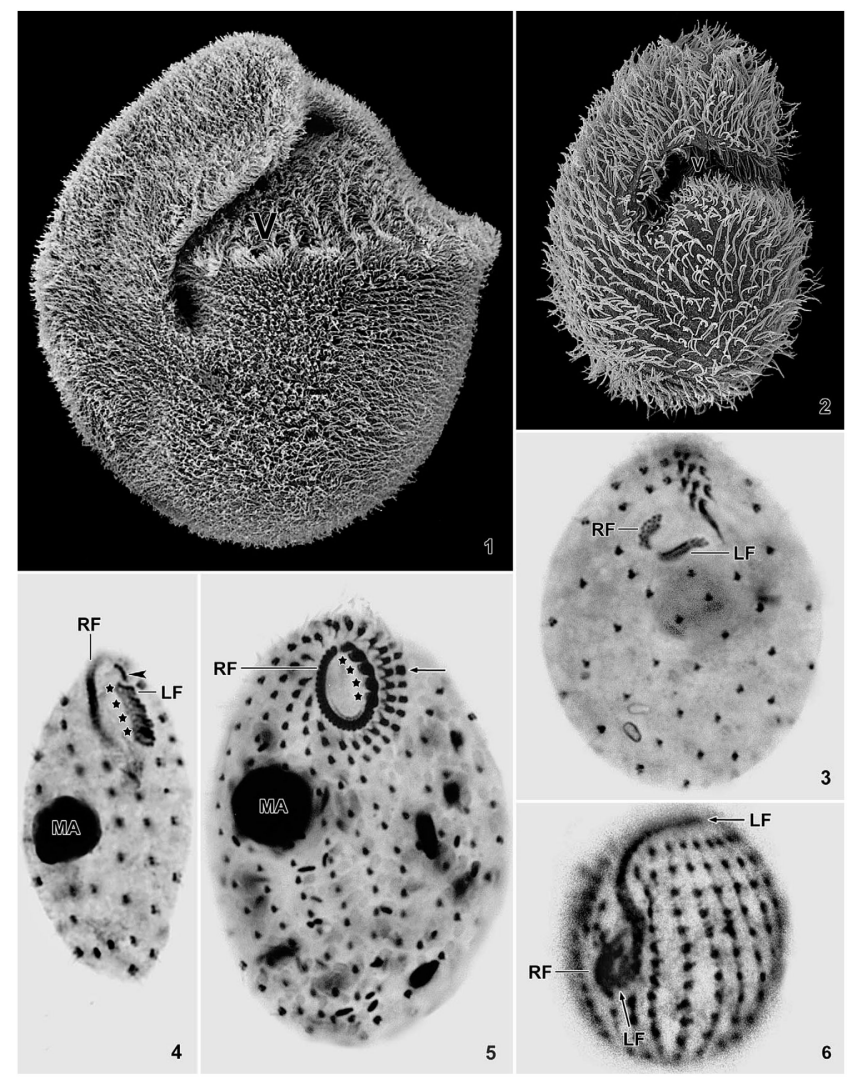

Figures 1-6.

Morphological variation within the Colpodea. Some of the species sequenced for the mtSSU-rDNA here are shown in scanning electron micrographs $(1,2)$ and in protargol silver preparations (3-6), exhibiting somatic and oral ciliary patterns. 1) Bresslauides discoideus and 2) Colpoda cucullus are closely related in the gene trees, but differ in overall size (up to $600 \mu \mathrm{m}$ vs. $150 \mu \mathrm{m}$ ), vestibulum (very large vs. small), oral ciliary structures (hausmanniellid vs. colpodid), and habit (predaceous vs. bacteriovorous). 3) Colpoda aspera and 6) Bardeliella pulchra are also closely related in the gene trees but with no node support, even though they are classified in different families (Colpodidae and Bardeliellidae). In $B$. pulchra (Fig. 6) the left oral ciliary field (LF) is greatly and uniquely modified to a very long, vertically oriented ribbon, while minute and horizontally oriented in C. aspera (Fig. 3), as in all other Colpoda species. 4) Cyrtolophosis mucicola and 5) Platyophrya bromelicola are in the Cyrtolophosidida I and II (Fig. 7), respectively. Cyrtolophosidida was originally characterized by a shared outer membrane of the micronucleus and macronucleus, a "simple" dikinetidal right oral ciliary field (RF), and a strand of brick-shaped adoral organelles in the left oral ciliary field (asterisks). The molecular data indicate that these features are either an ancestral state of the Colpodea, or evolved convergently at least twice. There are differences between the Cyrtolophosidida I and II: C. mucicola has a minute, vertically oriented organelle (Figure 4, arrowhead), while $P$. bromelicola has a membranelike ciliary condensation left of the adoral organelles (Figure 5, arrow). LF - left oral ciliary field, MA - macronucleus, RF - right oral ciliary field, V-vestibulum. 


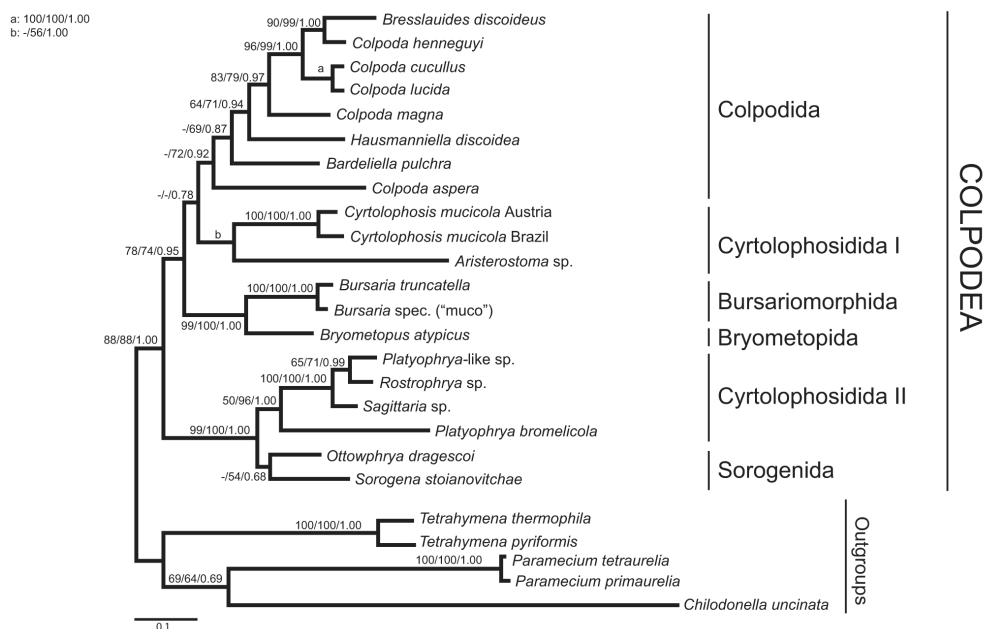

Figure 7.

Mitochondrial SSU-rDNA topology of the Colpodea. The most likely ML tree and its branch lengths are shown. The Bayesian tree inferred using MrBayes and the ML tree are identical in topology. Node support is as follows: MP bootstrap/ML bootstrap/BI (MrBayes) posterior probability. Support $<50 \%$ is shown as '-'. 

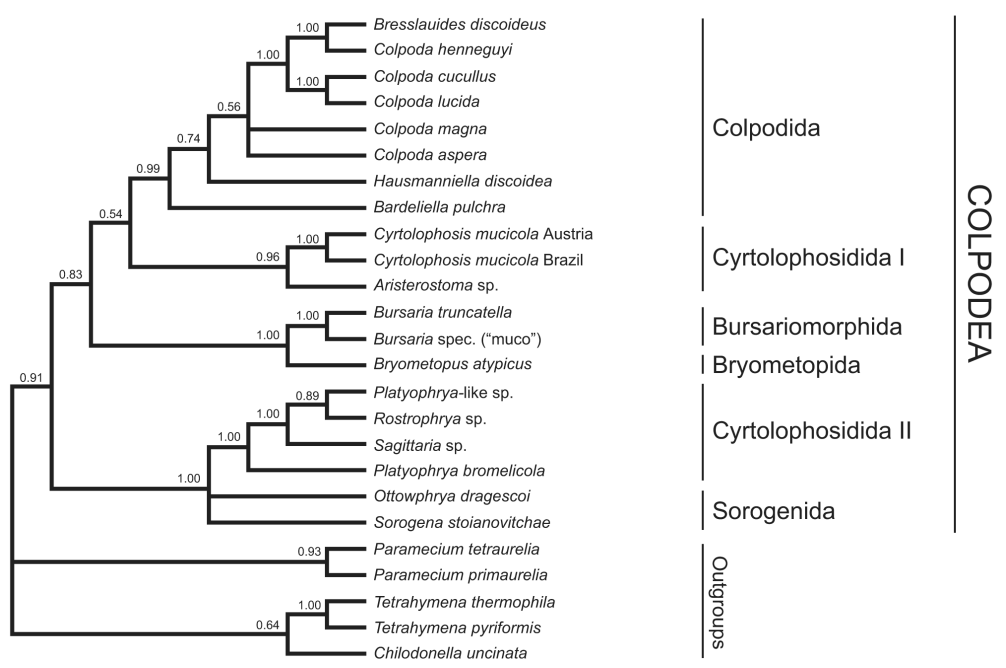

Figure 8.

Mitochondrial SSU-rDNA topology of the Colpodea. Bayesian tree and node support inferred using PhyloBayes. 


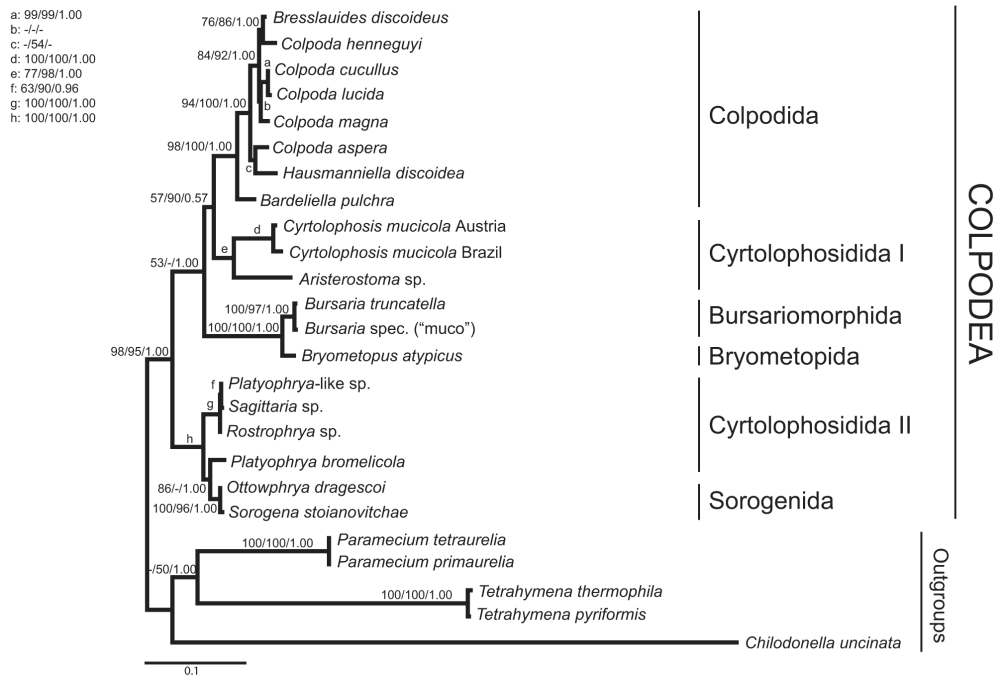

Figure 9.

Nuclear SSU-rDNA topology of the Colpodea. The most likely ML tree and its branch lengths are shown. The Bayesian tree inferred using MrBayes and the ML tree are identical in topology. Node support is as follows: MP bootstrap/ML bootstrap/BI (MrBayes) posterior probability. Support $<50 \%$ is shown as '-'. 


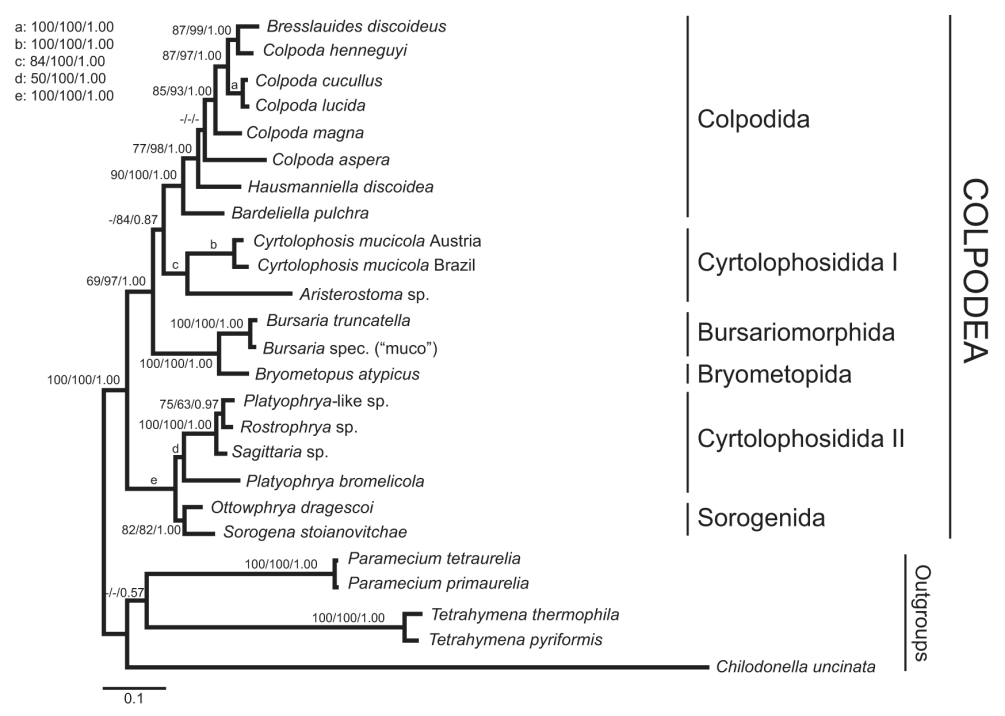

Figure 10.

Concatenated nuclear and mitochondrial SSU-rDNA topology of the Colpodea. The most likely ML tree and its branch lengths are shown. The Bayesian tree inferred using MrBayes and the ML tree are identical in topology. Node support is as follows: MP bootstrap/ML bootstrap/BI (MrBayes) posterior probability. Support $<50 \%$ is shown as '-'. 

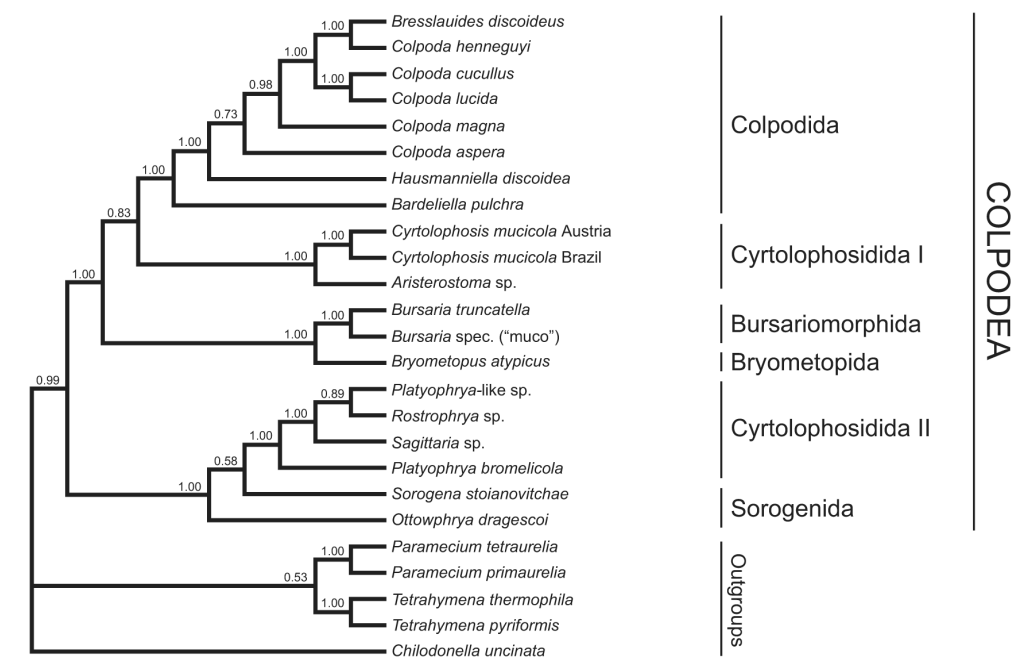

Figure 11.

Concatenated nuclear and mitochondrial SSU-rDNA topology of the Colpodea. Bayesian tree and node support inferred using PhyloBayes. 


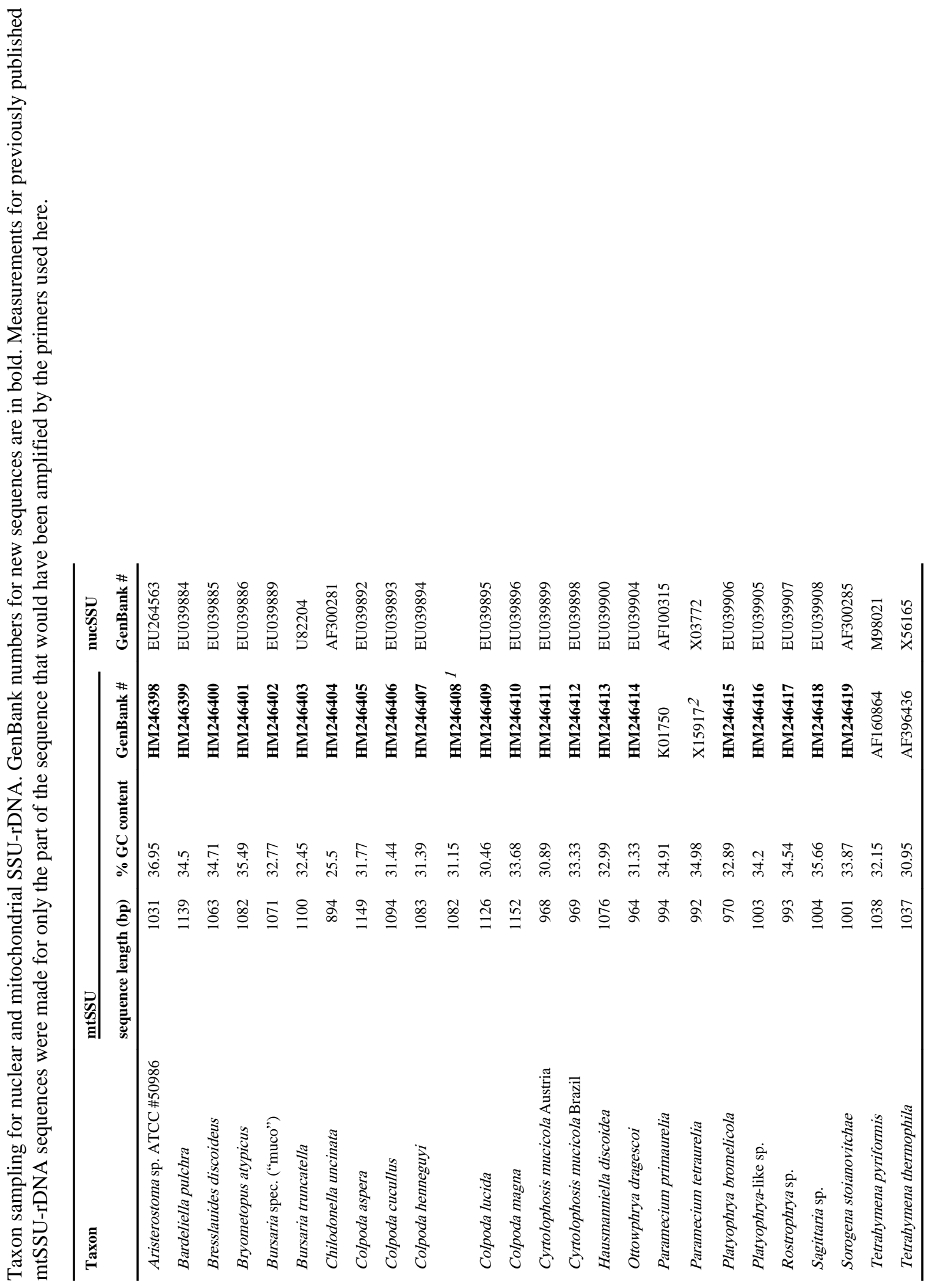




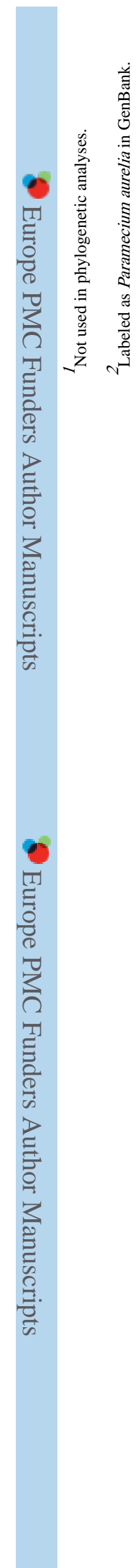


